\title{
Therapeutic Effect of Total Ginseng Saponin on Skin Wound Healing
}

\author{
Young Soo Kim ${ }^{1 \#}$, Ik-Hyun Cho ${ }^{2 \#}$, Moon-Jin Jeong ${ }^{3}$, Soon-Jeong Jeong ${ }^{3}$, Seung Yeol $\mathrm{Nah}^{4}$, \\ Young-Sik Cho ${ }^{5}$, Seung Hyun Kim ${ }^{1}$, Ara Go ${ }^{1}$, Se Eun Kim ${ }^{1}$, Seong Soo Kang ${ }^{1}$, Chang Jong Moon ${ }^{1}$, \\ Jong Choon Kim ${ }^{1}$, Sung Ho Kim ${ }^{1}$, and Chun Sik Bae ${ }^{1 *}$ \\ ${ }^{1}$ Department of Veterinary Surgery, Chonnam National University College of Veterinary Medicine, Gwangju 500-757, Korea \\ ${ }^{2}$ Department of Anatomy, College of Oriental Medicine and Institute of Oriental Medicine, Kyung Hee University, \\ Seoul 130-701, Korea \\ ${ }^{3}$ Department of Oral Histology and Developmental Biology, Chosun University School of Dentistry, Gwangju 501-759, Korea \\ ${ }^{4}$ Department of Physiology, Konkuk University College of Veterinary Medicine, Seoul 143-701, Korea \\ ${ }^{5}$ Department of Dental Hygiene, Namseoul University, Cheonan 331-707, Korea
}

In this study, we investigated the effects of total ginseng saponin (TGS) on the cutaneous wound healing process using histological analysis. A total of 24 ICR mice, 5-weeks-old, were used for all in vivo experiments. Mice were divided into control and TGS-treated groups and four equidistant $1-\mathrm{cm}$ full-thickness dorsal incisional wounds were created. The wounds were extracted at days 1, 3,5, and 7 post-injury for histomorphometrical analysis including wound area and contracture measurements, keratinocyte migration rate, and calculation of infiltrating inflammatory cells. The results showed that the wound area was smaller and keratinocyte migration rate was higher in the TGS-treated group than that of the control group from days 3 to 7 . Inflammatory cells in the TGS-treated group at days 1 and 3 were reduced compared to the control group. Wound contraction in the TGS-treated group was greater than in the control group on days 3 to 5 , and collagen deposition in the TGS-treated group was higher than in the control group during wound healing. The results indicate a beneficial effect of TGS when used to treat skin wounds.

Keywords: Panax ginseng, Skin wound healing, Total ginseng saponin, Mice

\section{INTRODUCTION}

The recovery process of skin wounds involves complex biological mechanisms, but can be basically classified into three phases: 1) inflammatory, 2) granulation formation as the result of cell proliferation, and 3) tissue remodeling [1]. These processes occur as a result of interactions between inflammatory cells, fibroblasts, and keratinocytes which induce microenvironmental changes at the wound site [2]. Tissue remodeling of the dermis involves collagen as well as antioxidants and a balance of matrix-producing proteins and protease enzymes es-

(cc) This is an Open Access article distributed under the terms of the Creative Commons Attribution Non-Commercial License (http://creativecommons.org/licenses/by-nc/3.0/) which permits unrestricted non-commercial use, distribution, and reproduction in any medium, provided the original work is properly cited. sential for the initiation of tissue remodeling during the recovery process of skin wounds [3].

Saponins are compounds extensively found in most plants which exist in a variety of types and are classified based on their internal structure [4]. Research has revealed that saponins can accelerate numerous biological activities including hemolytic [5,6], anti-bacterial $[7,8]$, anti-viral $[9,10]$, and anti-oxidative functions [11]. In addition, saponins reportedly have anti-inflammatory activity which can reduce edema and skin inflammation

Received 05 Jun. 2011, Revised 21 Jun. 2011, Accepted 21 Jun. 2011

"These two authors contributed equally to this work.

"Corresponding author

E-mail: csbae210@chonnam.ac.kr

Tel: +82-62-530-2876, Fax: +82-62-530-2809 
$[12,13]$. A saponin extracted from ginseng, known as ginsenoside, has been shown to accelerate neovascularization in burn wounds of the skin in mice, and increase vascular endothelial growth factor and interleukin (IL)$1 \beta$ which is one of the inflammatory cytokines known to induce the accumulation of macrophages at skin wound sites and accelerate wound healing [14]. However, according to previous studies, the recovery pattern of skin wounds caused by burns is reportedly quite different from that caused by physical injury or surgery [15]. Studies have reported some effects of saponins during the wound healing process; however, there is still insufficient information on surgical skin wounds. Thus, in this study, we produced surgical skin wounds and examined the effects of saponin during the wound healing process using histochemical and histomorphometric assessments.

\section{MATERIALS AND METHODS}

\section{Animals}

ICR mice ( $n=24$ ) weighing 45 to $55 \mathrm{~g}$ were purchased from Samtako (Osan, Korea). The mice were quarantined and acclimated for one week prior to use. The animals were housed in polycarbonate cages in a room maintained at $23 \pm 2^{\circ} \mathrm{C}$, a relative humidity of $50 \pm 5 \%$, artificial lighting from 08:00 to 20:00 h, and 13 to 18 air changes per hour. Tap water and commercial rodent chow (Samyang Feed, Daejeon, Korea) were provided ad libitum. The animals were blindly randomized into control $(n=12)$ and total ginseng saponin (TGS, $n=12$ ) groups.

\section{Animal model of incised skin wound}

Establishment of an animal model with an incised skin wound has been described previously [16]. Briefly, 6 -week-old male ICR mice ( $n=24)$, weighing 45 to $55 \mathrm{~g}$, were anesthetized by intraperitoneal injection of xylazine (Rompun ${ }^{\circledR}, 2 \mathrm{mg} / \mathrm{kg}$; Bayerkorea, Seoul, Korea) and zolazepam/tiletamine (Zoletil $50^{\circledR}, 0.1 \mathrm{mg} / \mathrm{kg}$; Virbac, Seoul, Korea). A scalpel was used to make four 1 -cm-long incisions on the central dorsum skin layer. Specimens $\left(1.5 \times 2 \mathrm{~cm}^{2}\right)$ were taken from the wound sites while anesthetized on days $1,3,5$, and 7 post-wounding ( $n=3 /$ group). Experiments conformed to the 'Principles of Laboratory Animal Care (National Institutes of Health publication no. 85-23, revised 1985)' and sought to minimize both the number of animals used and any suffering that might be experienced and were performed according to the Guidelines for the Care and Use of Laboratory Animals of Chonnam National University.

\section{Total ginseng saponin}

TGS was provided by the Korea Ginseng \& Tobacco Central Research Institute (Daejeon, Korea). The provided TGS contained 11 glycosides known as ginsenosides: $\mathrm{Rb}_{1}$ (15.82\%), $\mathrm{Rb}_{2}$ (7.79\%), Rc (8.06\%), Rd (7.57\%), Re (3.21\%), Rf (4.72\%), $\operatorname{Rg}_{1}(1.91 \%), \operatorname{Rg}_{2}(22.08 \%), \operatorname{Rg}_{3}$ (24.06\%), $\mathrm{Rh}_{1}(4.63 \%)$, and $\mathrm{Rh}_{2}(0.15 \%)$. The TGS (20 $\mathrm{mg} / \mathrm{mL}$ ) was dissolved in saline and administered once subcutaneously prior to making the surgical skin wound in the treated group.

\section{Histological analysis}

To compare changes between groups, mice were euthanized on days 1, 3, 5, and 7 post-wounding. Pictures of the wounds were taken with a digital camera (DMCFZ5GD; Panasonic, Kadoma, Japan) after attaching a ruler to the skin wound area. Skin samples from the wound area were taken and kept in $4 \%$ formaldehyde in phosphate-buffered saline (PBS, $\mathrm{pH} 7.4$ ) for $24 \mathrm{~h}$, then washed with PBS solution for $2 \mathrm{~h}$, dehydrated using graded alcohol, clarified, and finally embedded into paraffin. Sections, $6-\mu \mathrm{m}$-thick, were cut with a motorized rotary microtome MT990 (RMC Products, Tucson, AZ, USA). Hematoxylin and eosin (H\&E) staining was performed to measure the size of the wound area and detect any histomorphometric changes. To analyze the number of inflammatory cells, Giemsa staining was performed, and to analyze the collagen in the matrix formation, Masson's trichrome and picrosirius red staining were used. The wound area was measured the epithelium as well as the marginal area of the connective tissue. The number of inflammatory cells was counted at the left, right, and center of the wound area. The degree of keratinocyte migration was determined by measuring the size of the epithelial tissue rising from the edge of the wound, while wound contraction was calculated by measuring the distance between the edge of the wound and the damaged dermal layer. Stained tissue samples were observed using a polarizing microscope (Carl Zeiss, Goettingen, German), and the AxioVision LE release 4.6 (Carl Zeiss) image analysis program was used for all analysis.

\section{Statistical analysis}

Data presented are mean values from three animal \pm standard deviations. Statistical analysis of the data was performed using a Student's $t$-test with a Bonferroni correction for analysis of multiple comparisons. Differences were considered significant at $p<0.05$. 


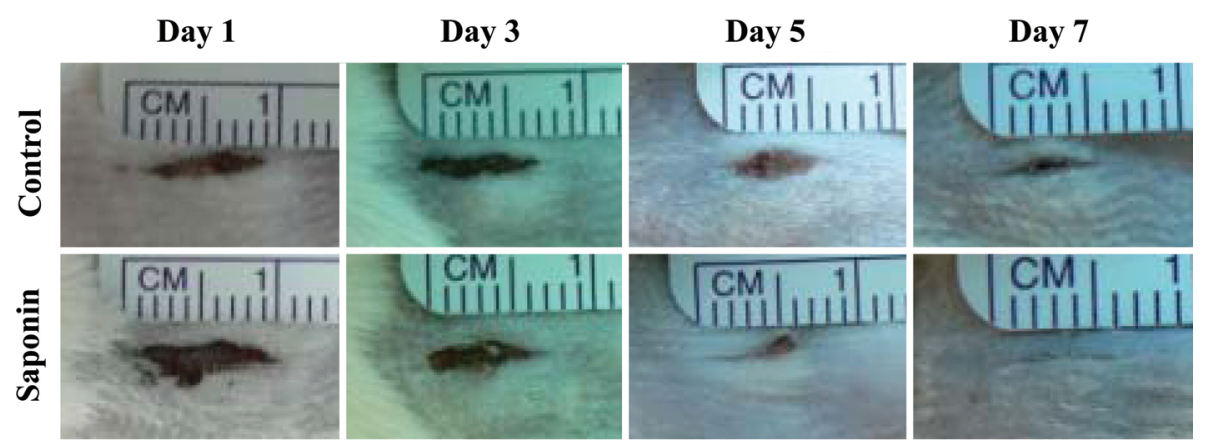

Fig. 1. Differences of dermal wound size between control and saponin-treated groups. Wound size was gradually reduced in both groups from day 1 to day 7 . In the saponin-treated group, the wound size was more reduced at days 5 and 7 compared to the control group.

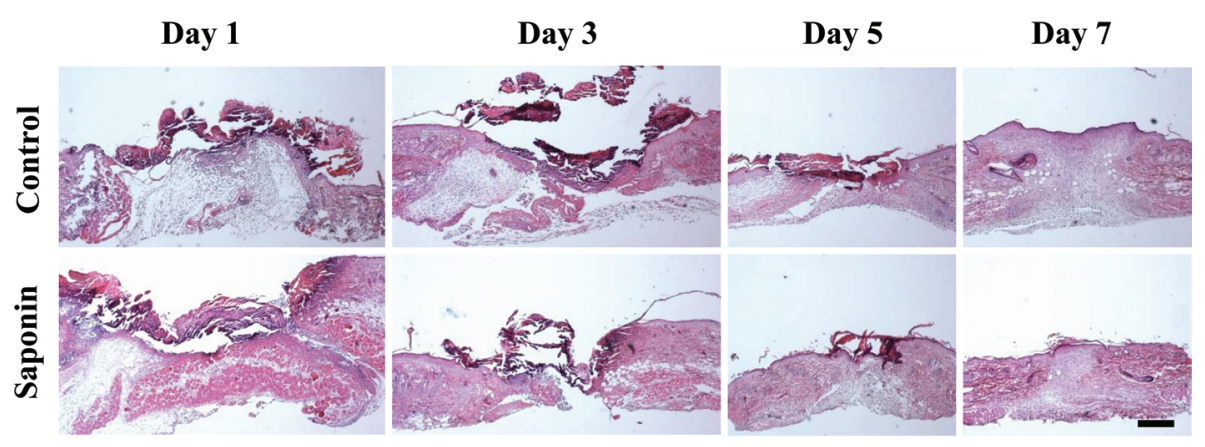

Fig. 2. Differences in wound area between control and saponin-treated groups during skin wound healing (H\&E, $\times 40)$. The wound area of the saponin-treated group was larger than the control group at day 1 but smaller at days 3-7. Scale bar $=200 \mu \mathrm{m}$.

\section{RESULTS}

\section{Wound healing tendencies after saponin treat- ment}

To examine the effect of saponin on the wound healing process, changes in wound size on days $1,3,5$, and 7 post-wounding were observed and compared between the untreated control group and the saponin-treated group. Wound size on day 5 in the saponin-treated group was much smaller compared to the control group, and by day 7 , both sides of the incision were confirmed to be completely joined in the treated group (Fig. 1).

\section{Histological and morphometric analysis of the skin wound healing following saponin treatment}

$\mathrm{H} \& \mathrm{E}$ staining was performed on wound tissue samples at various time points post-wounding. Most wound tissue appeared to have disintegrated in both the control and the saponin-treated groups, and scabs were observed. However, the amount of scab was smaller, epithelial cell migration was faster, and less residue in the dermal layer was found to exist in the saponin-treated group than in the control group. On day 5 post-wounding, epithelial cell migration was not complete in the control group, while in the saponin-treated group, it was almost complete and the amount of scab was less than in the control group. In addition, the amount of residue in the dermal layer in the saponin-treated group was confirmed to be much less compared to that of the control group. By day 7 , all scabs had come off in both groups. Epithelial cell migration was fully complete in the saponin-treated group but the wound contraction rate appeared to be higher (Fig. 2). Wound size tended to be reduced as time passed in both groups; however, by period, the wound sizes in the saponin-treated group were smaller than those of the control group during the wound healing process from days 3 to 7 (Fig. 3). Furthermore, while the keratinocyte migration rate was similar on day 1 and tended to increase in both groups as time passed, the rate in the saponin-treated group appeared to be higher than that of the control group (Fig. 4).

Histological analysis of inflammatory cells and
wound contraction rate during the skin wound
healing process
Giemsa staining was performed to analyze the number 


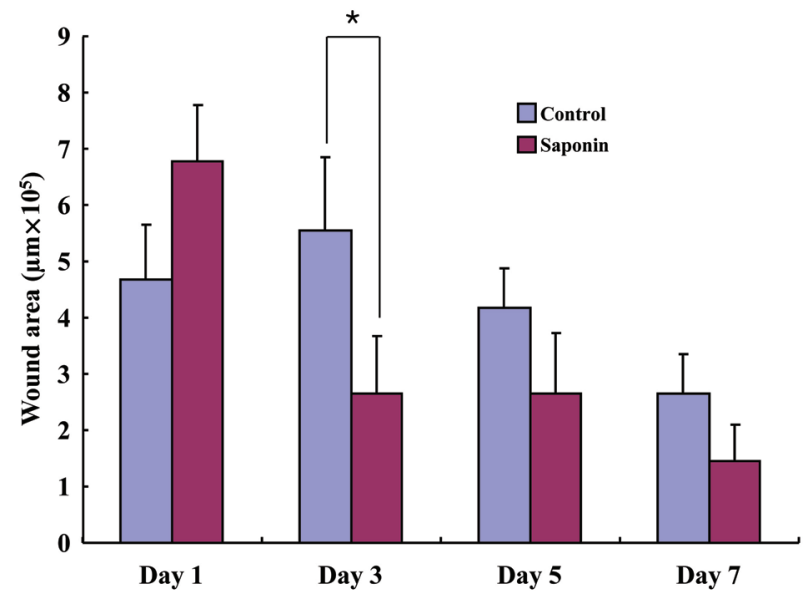

Fig. 3. The wound area of the saponin-treated group was larger than the control group at day 1 but smaller at days 3 to 7 . This result was established based upon morphometric analysis of wounds tissues from control and saponin-treated groups. ${ }^{*} p<0.05$.

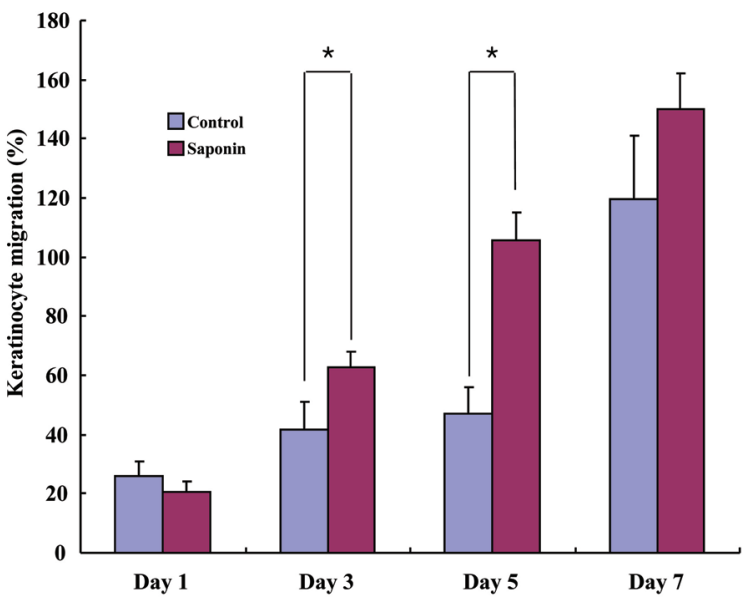

Fig. 4. Keratinocyte migration in wound tissue. The keratinocyte migration rate in the saponin-treated group was higher than that of the control group at days 3 to 7 . ${ }^{*} p<0.05$.

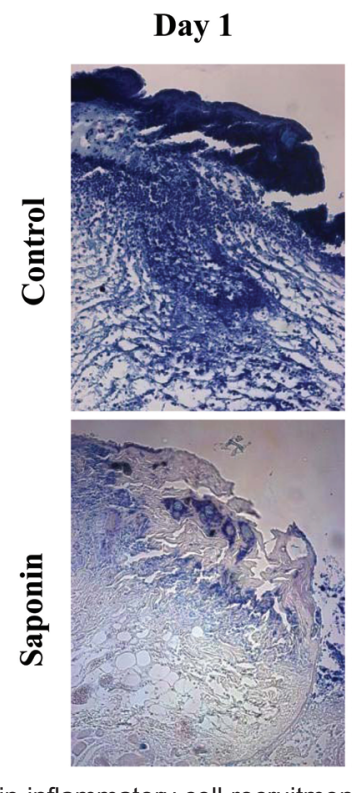

Day 3

Day 5
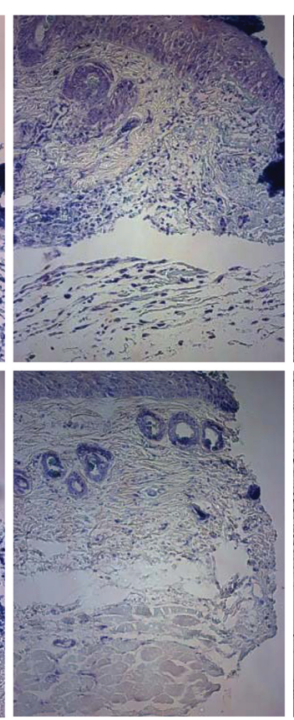

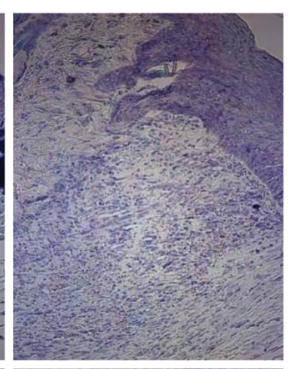

Day 7
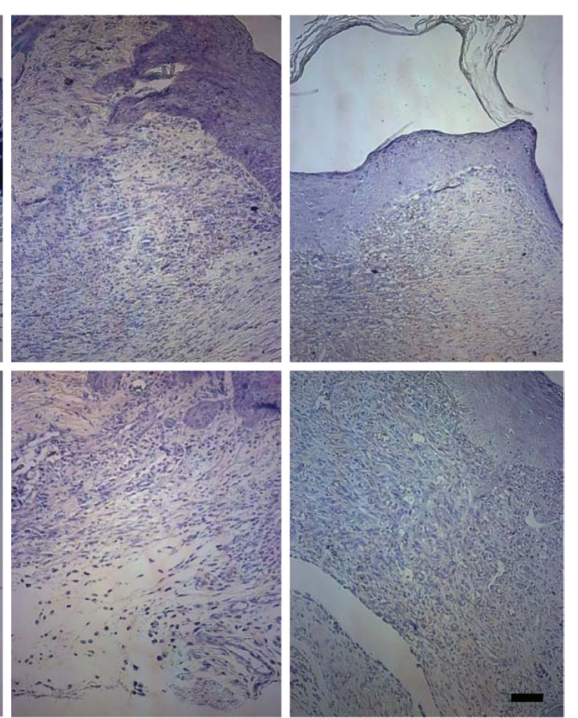

Fig. 5. Differences in inflammatory cell recruitment between control and saponin-treated skin wounds (Giemsa, $\times 400$ ). Inflammatory cells in the saponin-treated wounds were reduced from days 1 to 5 compared to control wound tissue, but increased at day 7 . All tissues were stained with Giemsa. Scale bar=20 $\mu \mathrm{m}$.

of inflammatory cells in the wounded area at different time periods. The largest cell numbers were seen on the first day post-wounding and gradually decreased over time. The number of inflammatory cells in the saponintreated group was noticeably smaller on days 1 and 3 compared to the control group and tended to dwindle over time (Fig. 5). However, the number of inflammatory cells in the saponin-treated group increased starting at day 5 and maintained until day 7 (Fig. 6). The control group showed an increase in the rate of wound contraction on day 7 after being at a constant level from days
1-5, while the saponin-treated group showed a gradual increase in the rate of wound contraction reaching the highest level on day 7. Specifically, the wound contraction rate of the saponin-treated group was lower than the control group on day 1 but began to increase from day 3 similar to the control group, eventually becoming higher than the control group on days 5 to 7 (Fig. 7).

\section{Analysis of substrate formation in the wound heal- ing process}

To confirm substrate formation in the dermis, Mas- 


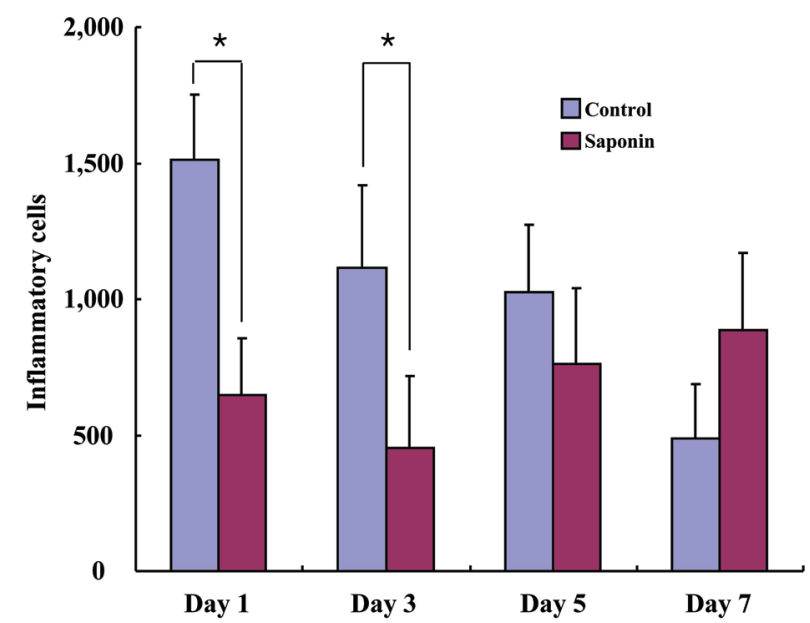

Fig. 6. Inflammatory cells count analysis of wound tissues. Inflammatory cells were counted in three distinctive areas (left, right, and middle of wound area). Inflammatory cells of the saponin-treated group were reduced compared with the control group at days 1 and 3 . However, at day 5 inflammatory cells of the saponin-treated group were increased compared to day 3 , and at day 7 inflammatory cells of the saponin-treated group were more increased than the control group. ${ }^{*} p<0.05$.

son's trichrome and picrosirius red staining were performed and the disposition time of collagen was calculated. In the control group, collagen synthesis stained a pale blue was identified around the wound from day 3 ,

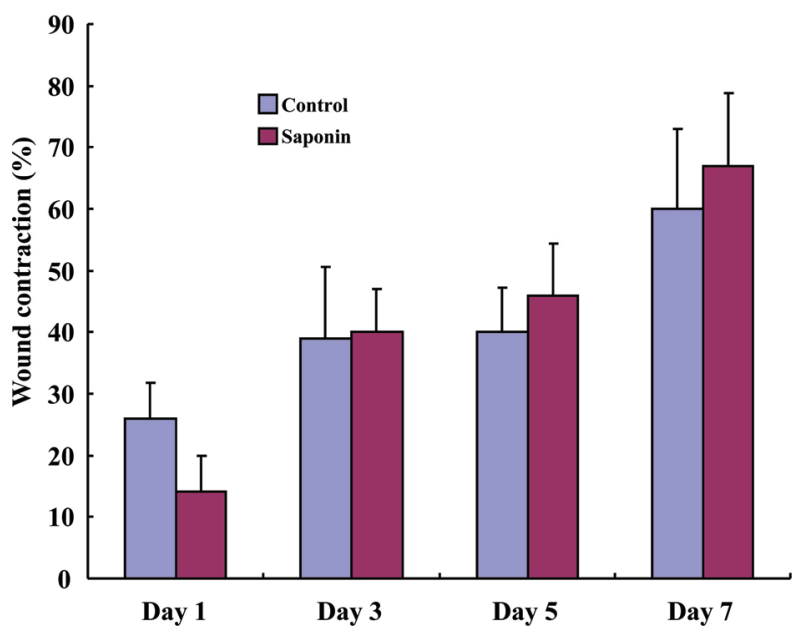

Fig. 7. Wound contraction measurements between control and saponin-treated groups. Wound contraction in the saponin-treated group was higher than that of the control group from day 3 to day 5 .

and tended to be active more or less in the tissues at days 5 and 7 (Fig. 8). As a result of confirmation of collagen disposition, collagen synthesis was first observed on day 5 and appeared to be increased on day 7. However, collagen synthesis was deeper in the saponin-treated group than the control group for the entire study period (Fig. 9).

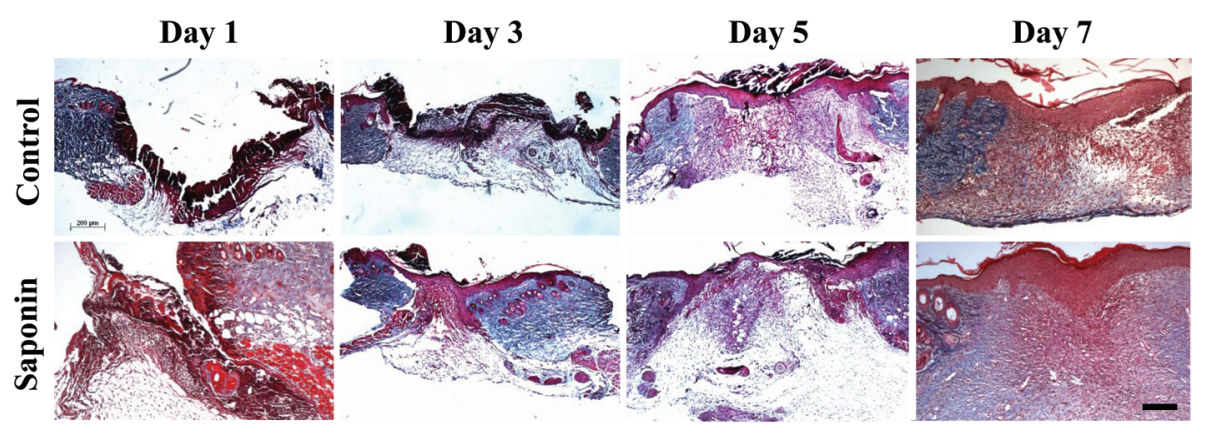

Fig. 8. Difference of accumulated collagen (blue color) in healing areas between control and saponin-treated groups (Masson's trichrome, $\times 40$ ). Collagen in the healing area of control and saponin-treated groups gradually increased from day 1 to day 7 . Aspects of collagen deposition in the saponin-treated group were greater than in the control group. Scale bar=100 $\mu \mathrm{m}$.
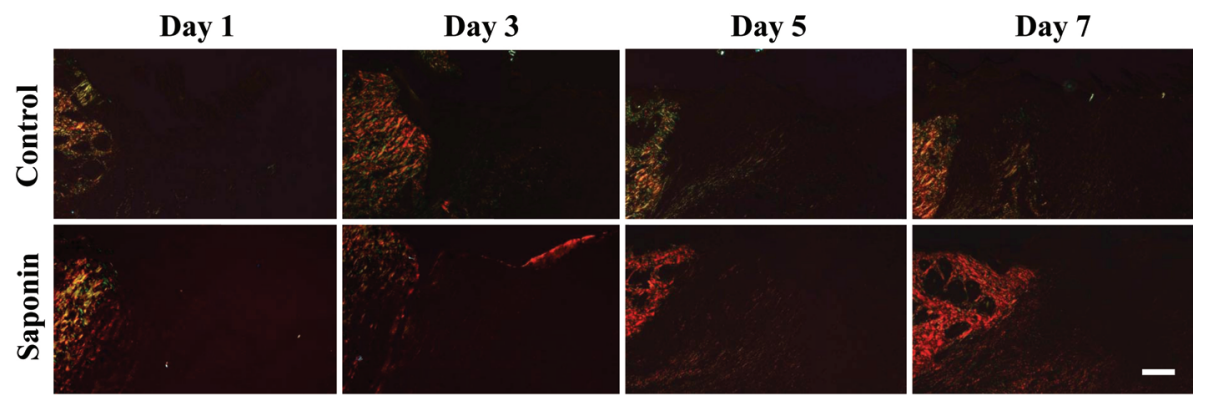

Fig. 9. Difference in accumulated collagen (bright red color) in the healing areas of control and saponin-treated groups (Picrosirius red, $\times 100)$. This result coincided with that of collagen staining. Scale bar=100 $\mu \mathrm{m}$. 


\section{DISCUSSION}

In mammals, wound healing involves a very prompt and efficient process in which bleeding from the wound is first stopped, then the damaged tissues are reformed, moisture is evaporated around the wound, and a functional defense membrane is formed to protect against microorganism penetration into the body. Skin wound healing can be classified into three phases which include the initial inflammatory reaction, re-epithelialization and granulation tissue formation, and finally tissue remodeling [17]. These classifications are based upon histological results or functional activities, but in fact, they overlap. For perfect healing to occur, interactions between cells and tissues which are deeply involved in these three phases are essential [18].

Blood coagulation is initiated by the formation of clots which form in combination with fibrin fiber bundles activated by blood coagulation factors and platelets. Fibronectin and vitronectin in blood plasma are included in the blood coagulation phase and temporarily form the substrate for cell migration. Keratinocytes migrate into this substrate and then proteinases work to form a scab around the wound. Growth factors and platelets secreted by the activated blood coagulation factors, complement components, and damaged cells, provoke the chemotactic stimulus [19]. While the blood coagulation process is in progress, neutrophils arrive first at the damaged tissue, within a few hours. The major function of the neutrophils is phagocytosis to eliminate infective agents at the site of damaged tissue, and provoke blood coagulation, inflammatory reaction, and healing activity by secreting various factors [20]. Next, monocytes arrive at the wound within two days and differentiate into macrophages, to perform phagocytosis and antigen presenting functions, secreting transforming growth factors- $\alpha$ and $\beta$, basic fibroblast growth factor, and platelet-derived growth factor to regulate the wound healing process [20].

Re-epithelialization and granulation tissue formation occur simultaneously within a few hours of the inflammatory reaction. Keratinocytes which exist around the edges of the wound and in residues of skin appendages begin to migrate into the wound and form a scab [19]. These keratinocytes are characteristically hyper-proliferative enabling them to fill the damaged epithelial layer and reform the basement membrane within two days after wound initiation, thereby restoring mutual cellular contacts. Through this process, keratinocytes differentiate to form the epidermal skin layer [19]. At almost the same time as fibroblasts located around the undamaged dermis begin to proliferate and migrate as a result of the stimulus caused by the aforementioned growth factors, granulation tissue formation is initiated [19]. Fibroblasts which migrate into the wound and proliferate synthesize proteoglycans, collagen type III, and collagen type I to reform the extracellular matrix around the wound [17]. Some fibroblasts differentiate into myofibroblasts and synthesize $\alpha$-smooth muscle actin, which provides mechanical tension to pull the edges of the wound to provoke contraction and eventually promote wound closure [21]. New blood vessels appear in granulation tissue as a result of migration and proliferation of endothelial cells, which is stimulated by macrophages or keratinocytes and growth factors secreted from endothelial cells [19]. The dermis remodeling phase of skin wound healing involves the full growth of the wound accompanied by collagenous scarring. This phase is characterized by a reduction of fibroblasts by apoptosis and removal of damaged blood vessels. The residual fibroblasts rearrange the collagen fiber to recover the original tension of the skin, repeating collagen deposition and degradation for several months; however, the original skin tension before being damaged can never be fully recovered $[19,22]$.

There are various medicines available to assist in skin wound healing, ranging from disinfectants such as ethyl alcohol, iodine, and ether, to ointments contained various antibiotics and steroid hormones, which can injure not only invading cells but also normal cells, as well as induce the emergence of resistant bacteria and cause various hypersensitivity reactions [23]. From ancient times, various natural substances have been widely used for wound healing, anti-aging, and the treatment of other diseases [24]. Among such extracts, the polyphenol compounds are known to be strong antioxidants capable of neutralizing free radicals by combining with active oxygen [24]. A hydroxyl group attaches to an aromatic ring of a polyphenol compound which combines with free radicals which appear during the metabolic process and form neutralized stable phenoxyl radicals [25]. Free radicals with which polyphenol compounds are combined are superoxide radical anions, hydroxyl radicals, lipid peroxyl radicals, nitric oxide radicals, and peroxynitrites. All of these have been known to play a significant role not only in the biological environment but also in the treatment of diseases [26].

Radicals produced by wounds are largely superoxide radical anions produced by neutrophils and macrophages, and also play an important role in mechanisms which remove microorganisms and pathogens [27]. Superoxide radical anions are quickly transformed into hydrogen 
peroxide $\left(\mathrm{H}_{2} \mathrm{O}_{2}\right)$ which is able to permeate microorganisms or pathogenic cell membranes by superoxide dismutase, promoting the formation of hypochlorous acid, chloramines, and aldehyde which are all maintained in more stable forms than $\mathrm{H}_{2} \mathrm{O}_{2}$, and are characterized by long half-lives. Thus, when $\mathrm{H}_{2} \mathrm{O}_{2}$ substances remain in the wound for extended periods, acute inflammatory reactions likely to damage even normal cells may occur [28].

Saponins are plant extracts endowed with extensive biological activities. The promotion of anti-oxidants and anti-inflammatory reactions are included among their functions [4]. Saponins are one of various types of glycosides existing in higher orders of plants [29]. Saponin types are classified and named based on internal structure. In particular, a certain saponin referred to as fruticesaponin B is known to have very high antiinflammatory activity [12]. Navarro et al. [13] reported that saponins may have high or low activity depending upon their internal structure. In fact, only two types of saponins tested reduced neutrophil entry to wounds thereby lessening chronic skin inflammatory reactions. In this study, the wound healing rate in the saponin-treated group progressed much farther than the control group at day 5 , and both sides of the incisional wounds was fully joined at day 7 . The wound area shrunk more in the saponin-treated group than the control group during all time periods evaluated with the exception of day 1 , and the rate of keratin cell migration in the saponin-treated group appeared to be higher than the control group during all periods except day 1 . Other research reported that the burn wound area in a saponin-treated group gradually increased up to day 4 then gradually decreased until day 20 , while, in a control group, the burn wound area gradually increased up to day 8 and then tended to diminish in size [14]. It is assumed in this case that the long lead time in healing the burn wound was the result of an inflammatory reaction around the burn wound which persisted longer [30]. In addition, it was reported that saponin increased the expression of factors relevant to proliferation, and consequently, promoted the proliferation of epidermal cells [31]. Likewise, in our study, we found the rate of keratin cell migration involved in re-epithelialization to be faster in the saponin-treated group than in the control group. Therefore, it is assumed that saponin not only enhances epidermal cell proliferation but also promotes keratin cell migration. When the influx of inflammatory cells was measured after wounding, the number of cells in the saponin-treated group tended to be obviously less and diminish at day 1 and day 3 compared to the control group, but appeared to have a tendency to be increased from day 5 , and eventually the number of inflammatory cells appeared to be greater in the treated group than the control group at day 7. Even in the study by Kimura et al. [14], the number of leukocytes and macrophages appeared to increase up to day 9 after a burn wound, reportedly due to the expression of IL- $1 \beta$ by hypoxiainducible factor- $1 \alpha$ which induced the accumulation of macrophages. Accordingly, it is assumed that saponin was involved in the inhibition of the inflammatory reaction at the early stage. Furthermore, wound shrinkage measurements appeared to be sharply increased from day 3 on. Matrix remodeling analysis confirmed that matrix synthesis was promoted in the saponin-treated group compared to the control group. A recent study revealed that when saponin was used to treat skin tissue exposed to ultraviolet rays, collagen synthesis of fibroblasts was increased and expression of matrix metalloproteinases was inhibited [32]. Furthermore, it was also revealed that saponin increased collagen synthesis in skin fibroblasts through phosphorylation of Smad 2 protein [33]. Thus, it is assumed that saponin will promote the re-synthesis of matrix at the site of a skin wound.

These findings indicate that saponin not only promotes re-epithelialization of the wound but also effectively inhibits inflammatory reactions during the early phase, and promotes matrix synthesis throughout the wound healing process. Based upon this information, saponin is beneficial in healing incisional skin wounds.

\section{ACKNOWLEDGEMENTS}

This study was financially supported by Chonnam National University, 2009.

\section{REFERENCES}

1. Stephens P, Thomas DW. The cellular proliferative phase of the wound repair process. J Wound Care 2002;11:253261.

2. Werner S, Krieg T, Smola H. Keratinocyte-fibroblast interactions in wound healing. J Invest Dermatol 2007; 127:998-1008.

3. Blazso G, Gabor M, Schonlau F, Rohdewald P. Pycnogenol accelerates wound healing and reduces scar formation. Phytother Res 2004;18:579-581.

4. Guclu-Ustundag O, Mazza G. Saponins: properties, applications and processing. Crit Rev Food Sci Nutr 2007;47:231-258.

5. Baumann E, Stoya G, Volkner A, Richter W, Lemke 
C, Linss W. Hemolysis of human erythrocytes with saponin affects the membrane structure. Acta Histochem 2000;102:21-35.

6. Oda K, Matsuda H, Murakami T, Katayama S, Ohgitani T, Yoshikawa M. Adjuvant and haemolytic activities of 47 saponins derived from medicinal and food plants. Biol Chem 2000;38:67-74.

7. Killeen GF, Madigan CA, Connolly CR, Walsh GA, Clark C, Hynes MJ, Timmins BF, James P, Headon DR, Power RF. Antimicrobial saponins of Yucca schidigera and the implications of their in vitro properties for their in vivo impact. J Agric Food Chem 1998;46:3178-3186.

8. Konishi M, Hano Y, Takayama M, Nomura T, Hamzah AS, Ahmad RB, Jamani H. Triterpenoid saponins from Hedyotis nudicaulis. Phytochemistry 1998;48:525-528.

9. Simoes CM, Amoros M, Girre L. Mechanism of antiviral activity of triterpenoid saponins. Phytother Res 1999; 13:323-328.

10. Apers S, Baronikova S, Sindambiwe JB, Witvrouw M, De Clercq E, Vanden Berghe D, Van Marck E, Vlietinck A, Pieters L. Antiviral, haemolytic and molluscicidal activities of triterpenoid saponins from Maesa lanceolata: establishment of structure-activity relationships. Planta Med 2001;67:528-532.

11. Yogeeswari P, Sriram D. Betulinic acid and its derivatives: a review on their biological properties. Curr Med Chem 2005;12:657-666.

12. Just MJ, Recio MC, Giner RM, Cuéllar MJ, Máñez S, Bilia AR, Ríos JL. Anti-inflammatory activity of unusual lupane saponins from Bupleurum fruticescens. Planta Med 1998;64:404-407.

13. Navarro P, Giner RM, Recio MC, Manez S, CerdaNicolas M, Ríos JL. In vivo anti-inflammatory activity of saponins from Bupleurum rotundifolium. Life Sci 2001;68:1199-1206.

14. Kimura Y, Sumiyoshi M, Kawahira K, Sakanaka M. Effects of ginseng saponins isolated from red ginseng roots on burn wound healing in mice. Br J Pharmacol 2006;148:860-870.

15. Wang ZY, Zhang J, Lu SL. Objective evaluation of burn and post-surgical scars and the accuracy of subjective scar type judgment. Chin Med J (Engl) 2008;121:2517-2520.

16. Guan DW, Ohshima T, Kondo T. Immunohistochemical study on Fas and Fas ligand in skin wound healing. Histochem J 2000;32:85-91.

17. Clark RA. Wound repair: overview and general considerations. In: Clark RA, Henson PM. The molecular and cel- lular biology of wound repair. 2nd ed. New York: Plenum Press, 1996. p.513-560.

18. Toriseva M, Kahari VM. Proteinases in cutaneous wound healing. Cell Mol Life Sci 2009;66:203-224.

19. Singer AJ, Clark RA. Cutaneous wound healing. N Engl J Med 1999;341:738-746.

20. Eming SA, Krieg T, Davidson JM. Inflammation in wound repair: molecular and cellular mechanisms. J Invest Dermatol 2007;127:514-525.

21. Hinz B, Mastrangelo D, Iselin CE, Chaponnier C, Gabbiani G. Mechanical tension controls granulation tissue contractile activity and myofibroblast differentiation. Am J Pathol 2001;159:1009-1020.

22. Hinz B. Formation and function of the myofibroblast during tissue repair. J Invest Dermatol 2007;127:526-537.

23. Kang JS. Cosmetic surgery. 3rd ed. Seoul: Kunga Press, 2004.

24. Hsu S. Green tea and the skin. J Am Acad Dermatol 2005;52:1049-1059.

25. Rice-Evans CA, Miller NJ, Paganga G. Structure-antioxidant activity relationships of flavonoids and phenolic acids. Free Radic Biol Med 1996;20:933-956.

26. Packer L, Rimbach G, Virgili F. Antioxidant activity and biologic properties of a procyanidin-rich extract from pine (Pinus maritima) bark, pycnogenol. Free Radic Biol Med 1999;27:704-724.

27. Babior BM. Phagocytes and oxidative stress. Am J Med 2000;109:33-44.

28. Sen CK, Khanna S, Gordillo G, Bagchi D, Bagchi M, Roy S. Oxygen, oxidants, and antioxidants in wound healing: an emerging paradigm. Ann N Y Acad Sci 2002;957:239249.

29. Sparg SG, Light ME, van Staden J. Biological activities and distribution of plant saponins. J Ethnopharmacol 2004;94:219-243.

30. Ramzy PI, Barret JP, Herndon DN. Thermal injury. Crit Care Clin 1999;15:333-352, ix.

31. Choi S. Epidermis proliferative effect of the Panax ginseng ginsenoside $\mathrm{Rb}_{2}$. Arch Pharm Res 2002;25:71-76.

32. Kim YG, Sumiyoshi M, Sakanaka M, Kimura Y. Effects of ginseng saponins isolated from red ginseng on ultraviolet B-induced skin aging in hairless mice. Eur J Pharmacol 2009;602:148-156.

33. Lee J, Jung E, Lee J, Huh S, Kim J, Park M, So J, Ham Y, Jung K, Hyun CG et al. Panax ginseng induces human type I collagen synthesis through activation of Smad signaling. J Ethnopharmacol 2007;109:29-34. 